\title{
Photon Green's functions theory for Coulomb correlated quantum well lasers
}

\author{
M.F. Pereira Jr. * \\ Instituto de Fisica, Universidade Federal da Bahia, 40210-340, Salvador BA Brazil
}

\begin{abstract}
A photon Green's function theory is used to incorporate Bethe-Salpeter-like many body corrections in the computations of output spectra of semiconductor quantum well lasers. Coulomb, quantum-confinement, multiple valence band coupling and cavity resonator effects are consistently included in the theory. Numerical results are presented for multiple quantum wells, under different design, and excitation conditions.
\end{abstract}

Keywords: semiconductor lasers; quantum wells, many body effects, Green's functions.

Paper received 28.01.04; accepted for publication 17.06.04.

\section{Introduction}

The simulation of semiconductor lasers is a fascinating challenge to modern physics [1]. They operate in a highly excited regime, where many-particle effects play a dominant role [2].

A previous approach for quantum well lasers has successfully described the combination of resonant cavity, band structure and many body effects in a temperature regime and for materials where a vertex-type of approximation still successfully describes the excited media [3]. There are however experimental conditions, especially at low temperatures where higher order Coulomb corrections must be dealt with $[4,5]$.

In this paper, we present solutions of a Bethe-Salpeter type of equation for quantum wells within a photon Green's functions approach capable of handling the resonant cavity microscopically, as well as quantum confinement and band structure effects. The microscopic approach consistently describes Pauli-blocking, the screening of the Coulomb interaction, Coulomb enhancement of the polarization function, and band gap shrinkage.

The many-body approach used here is based on Keldysh Green's functions for carriers $(G)$, photons $(D)$, and plasmons $(W)$ to describe the coupled light-excited semiconductor system $[3,6]$. Here, we just outline the method with words and a few representative equations.

\section{Main equations}

The Keldysh Green's function's time evolution is described by Dyson equations, characterized by free propagators $G_{0}^{-1}, D_{0}{ }^{-1}, W_{0}{ }^{-1}$, and selfenergies, $S, P$, and $p$, being the carrier selfenergy, the transverse, and the longitudinal polarization functions, respectively.

Detailed band-structure and quantum-confinement effects, obtained by solving the Luttinger Hamiltonian, are included in the free-carrier propagator $G_{0}{ }^{-1}$, and serve as the input for the solution of our many-body problem. Each of the selfenergies changes the bare into dressed propagators in a specific way.

The transverse polarization, $P$ describes the optical response of the system, and can be written as a sum of an RPA term and a Coulomb-correlation contribution, expressed by the solutions of the Bethe-Salpeter Eq. (4),

$$
P(1,1,2,2)=G(1,2) G(2,1)+G(1,3) G(4,1) P(3,4,2,2) .
$$

In this paper, we consider Coulomb correlations beyond RPA in the spectral density of photons but restrict the carrier selfenergies to RPA, i.e., $\Sigma(1,2)=G(1,2) W(1,2)$.

In order to describe light emission, we refer to the quantum mechanical Poynting vector,

$$
\langle\vec{S}(1)\rangle=\frac{c}{8 \pi}\langle\vec{E}(1) \times \vec{B}(2)-\vec{B}(2) \times \vec{E}(1)\rangle_{1=2} \approx D(12)_{1=2},(2)
$$




\section{M.F. Pereira Jr.: Photon Green's functions theory for Coulomb correlated quantum well lasers}

where D is the photon Green's function introduced above. For linearly polarized light, the expression reduces to

$$
\begin{aligned}
& \left\langle S_{x}\left(\vec{R}_{1}\right)\right\rangle=\int^{\infty} I(\omega) d \omega, \\
& I(\omega)=\frac{\hbar \omega^{0}}{4 \pi^{2} c^{3}} \tan ^{2} \theta i P^{<}(\omega) L F_{L}(\omega),
\end{aligned}
$$

where и is a small aperture angle characterizing paraxial propagation, and the mode structure is characterized by the cavity function,

$$
F_{L}(\omega)=\frac{1}{\left|1-r^{2}\right|^{2}} \frac{1}{|n(\omega)|^{2}} \frac{1}{|1+n(\omega)|^{2}} e^{-\frac{\omega}{c} L n_{2}(\omega)} J_{L}(\omega)
$$

with the slowly varying form factor and complex propagation wavenumber given respectively by

$J_{L}(\omega)=\left(1+|r|^{2}\right) \frac{\sinh \left(q_{2} L\right)}{q_{2} L}+\left(r^{*}+r\right) \frac{\sin \left(q_{1} L\right)}{q_{1} L}$,

and $q^{2}=\frac{\omega^{2} n^{2}}{c^{2}}, n_{2} \approx-\frac{c^{2}}{2 \omega^{2} \sqrt{\varepsilon(\infty)}} \mathfrak{I} P^{r}(\omega)$, with $n_{1}(\omega)$ obtained by a Kramers-Kronig transformation.

The polarization function satisfies the Kubo-MartinSchwinger (KMS) sum rule [2,4].

So, once $\mathfrak{S} P^{r}(\omega)$ is computed, the carrier recombination spectra is immediately obtained, ( $\mu$ is the total, electron + hole, chemical potential),

$$
P^{<}(\omega)=\frac{-2 i \mathfrak{I} P^{r}(\omega)}{1-\exp (\beta(\hbar \omega-\mu))} .
$$

\section{Numerical results and discussion}

Figure 1 depicts the TE absorption/gain of $3 \mathrm{~nm} \mathrm{ZnCdSe/}$ ZnSSe quantum well at 50K. From top to bottom, the carrier densities are $N=0,0.555,1.11,2.22 \cdot 10^{18}$ carriers $/ \mathrm{cm}^{3}$. Figure 2, is similar to Fig. 1, but for $15 \mathrm{~nm} \mathrm{ZnSe} \mathrm{/} \mathrm{Zn}, \mathrm{Mg})$ $(\mathrm{S}, \mathrm{Se}) /(\mathrm{Zn}, \mathrm{Mg})(\mathrm{S}, \mathrm{Se})$ quantum well at $200 \mathrm{~K}$. From top to bottom, the carrier densities are $N=0,0.01,0.05,0.1,0.5,1.0$, $1.5,2.0,2.5,3.0,4.0,5.0$, and $6.0 \cdot 10^{18}$ carriers $/ \mathrm{cm}^{3}$.

Figure 3 shows an application for the TE emission spectra of the quantum well of Figure 2, with $21 \mathrm{~nm}$ barriers and total resonator lengths from left to right, $L=60,80,90$, $123 \mu \mathrm{m}$. The carrier density is fixed at $N=3 \cdot 10^{18}$ carriers $/ \mathrm{cm}^{3}$.

The spectra are normalized to 1 , but as the resonator length used in the computation increases, the gain equal to loss condition is reached and the output increases and evolves from multi-mode to essentially mono-mode.

The idea is to illustrate the change from multi-to single-mode operation, which in practice would happen for a fixed resonator length in a given structure as the density of carriers in the active region increases, and the gain equal loss condition is approached. The scaling ratios with respect to the output at $L=123 \mu \mathrm{m}$ are $I_{\max , L}=123 / I_{\max , L}=60=534, I_{\max , L}=123 / I_{\max , L}=80=231$, $I_{\max , L}=123 / I_{\max , L}=90=95$.

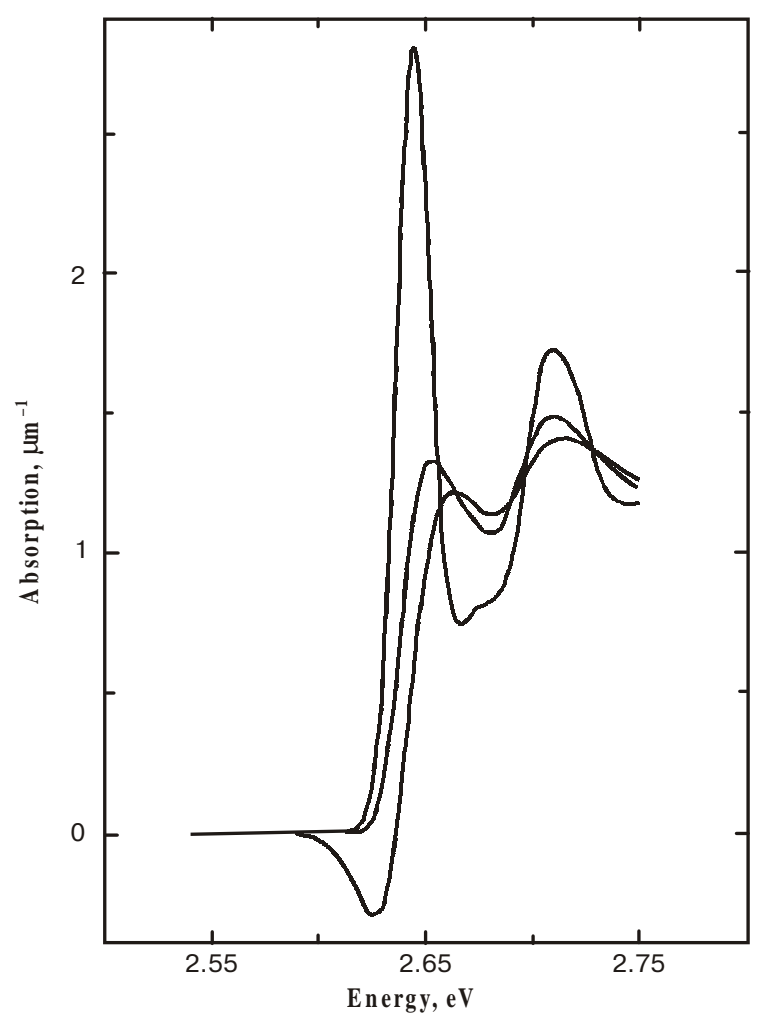

Fig. 1. TE absorption/gain of a $3 \mathrm{~nm} \mathrm{ZnCdSe/ZnSSe} \mathrm{quantum} \mathrm{well}$ at $50 \mathrm{~K}$. From top to bottom, the carrier densities are $N=0,0.555$, $1.11,2.22 \cdot 10^{18}$ carriers $/ \mathrm{cm}^{3}$.

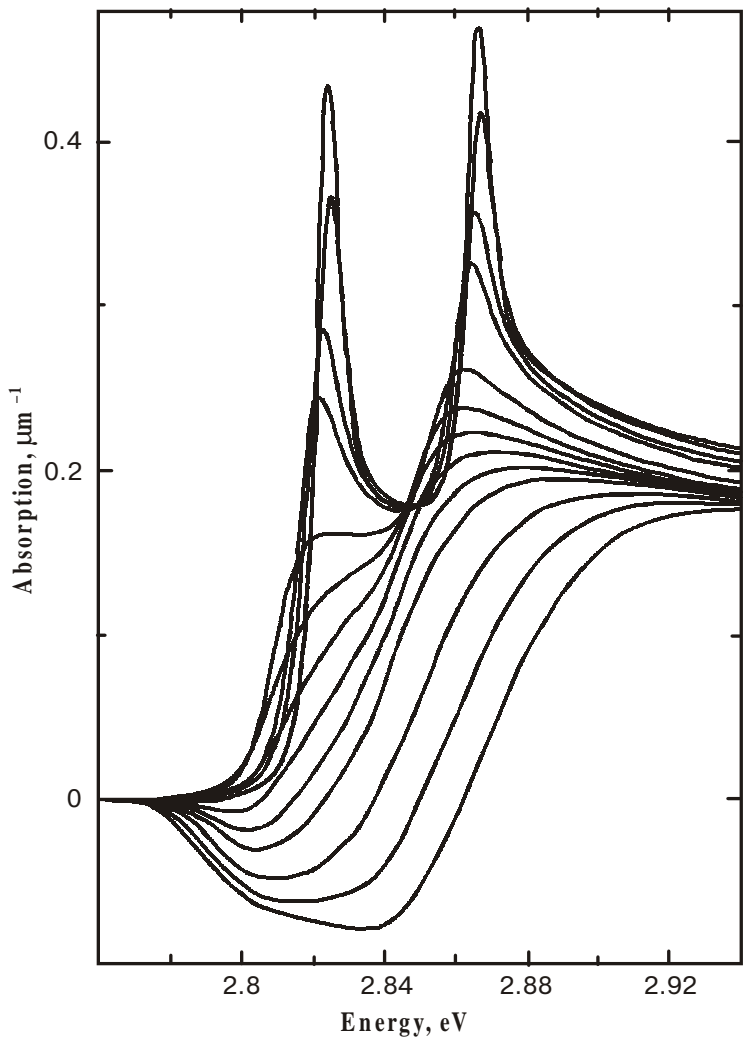

Fig. 2. TE absorption/gain of $15 \mathrm{~nm} \mathrm{ZnSe} /(\mathrm{Zn}, \mathrm{Mg})(\mathrm{S}, \mathrm{Se}) /$ / $(\mathrm{Zn}, \mathrm{Mg})(\mathrm{S}, \mathrm{Se})$ quantum well at $200 \mathrm{~K}$. From top to bottom, the carrier densities are $N=0,0.01,0.05,0.1,0.5,1.0,1.5,2.0,2.5$, $3.0,4.0,5.0$, and $6.0 \cdot 10^{18}$ carriers $/ \mathrm{cm}^{3}$. 


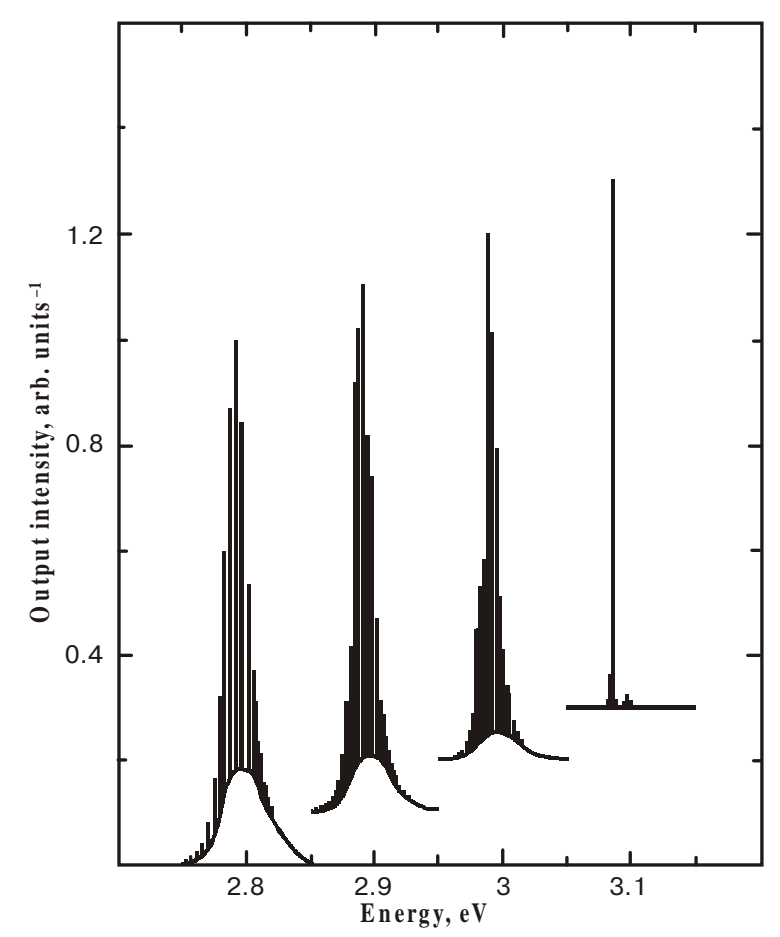

Fig. 3. TE emission spectra of the quantum well of Figure 2, with $21 \mathrm{~nm}$ barriers and total resonator lengths from left to right, $L=60$, $80,90,123 \mu \mathrm{m}$. The carrier density is fixed at $N=3 \cdot 10^{18}$ carriers $/ \mathrm{cm}^{3}$. The scaling ratios with respect to the output at $L=123 \mathrm{~mm}$ are $I_{\max , L}=123 / I_{\max , L}=60=534, I_{\max , L}=123 / I_{\max , L}=80=231, I_{\max , L}=123 /$ $/ I_{\max , L=90}=95$. The curves are displaced in the units of the plot, from left to right, respectively by $(0,0),(0.1,0.10),(0.2,0.2)$, $(0.3,0.3)$.

Furthermore, in order to obtain a clearer plot, the curves are displaced in the units of the plot, from left to right, respectively by $(0,0),(0.1,0.10),(0.2,0.2)$, $(0.3,0.3)$. In this case, the corresponding version of the vertex corrections of Ref. [3] cannot be used, since as depicted in Figs 1 and 2, the pronounced exciton-like bumps in the absorption spectra are a clear evidence of strong Coulomb correlations, which cannot be described by vertex corrections only.

In both cases, $T$-matrix correlations beyond RPA are included in the spectral density of photons, but the carrier self-energies are restricted to RPA, with the same level of approximations discussed in Ref. [4].

The Coulomb correlations are so strong that optical gain co-exists with features, which are well described by a strongly interacting electron-hole plasma. This interpretation is consistent with recent experimental results [5].

In summary, the approach presented here allows for the description of optical properties of semiconductor lasers using a Green's functions technique. It has the advantage of describing cavity and many body effects in a consistent way.

Although all the results presented are for steady state, the general theory outlined is valid for general nonequilibrium conditions. The approach has the further advantage of allowing the inclusion of high order correlation effects consistently. Laser output spectra are presented for different cavity, and excitation conditions in the presence of strong Coulomb correlations.

Although low-dimensional II-VI semiconductors have been used, the method is useful in the description of other dielectric materials, relevant for basic and applied physics alike.

\section{Acknowledgments}

Research supported by Conselho Nacional de Pesquisas, CNPq of Brazil.

\section{References}

1. P. Zory, Quantum Well Lasers, Academic Press, San Diego, 1993.

2. R. Zimmermann, Many-Particle Theory of Highly Excited Semiconductors, "Teubner Texte zur Physik", Leipzig (1987).

3. M.F. Pereira Jr., and K. Henneberger// Phys. Rev. B, 53, p. 16485 (1996).

4. M.F. Pereira Jr., and K. Henneberger // Phys. Rev. B, 58, p. 2064 (1998).

5. P. Michler, M.Vehse, J. Gutowski, M. Behringer, D. Hommel, M.F. Pereira Jr., and K. Henneberger // Phys. Rev. B, 58, p. 2055 (1998).

6. L.V. Keldysh // Zh. Eksp. Teor. Fiz., 20(4) (1965). 